\title{
Correction to: Good polynomials for optimal LRC of low locality
}

\author{
Ruikai Chen $^{1,2} \cdot$ Sihem Mesnager $2,3,4 \cdot$ Chang-An Zhao ${ }^{1,5,6_{(D}}$
}

Accepted: 24 June 2021 / Published online: 13 July 2021

(c) Springer Science+Business Media, LLC, part of Springer Nature 2021

\section{Correction to: Des. Codes Cryptogr. (2021) 89:1639-1660 https://doi.org/10.1007/s10623-021-00886-4}

In this article, several affiliations were incorrect: the affiliation for the author Ruikai Chen should have been Affiliation 1 and 2. i.e., 1. School of Mathematics, Sun Yat-sen University, Guangzhou 510275, People's Republic of China and 2. Department of Mathematics, University of Paris VIII, 93526 Saint-Denis, France; author Chang-An Zhao should have been Affiliations 1, 5 and 6. i.e., 1. School of Mathematics, Sun Yat-sen University, Guangzhou 510275, People's Republic of China, 5. State Key Laboratory of Information Security, Institute of Information Engineering, Chinese Academy of Sciences, Beijing 100093, People's Republic of China and 6. Guangdong Key Laboratory of Information Security, Guangzhou 510006, People's Republic of China.

The original article has been corrected.

Publisher's Note Springer Nature remains neutral with regard to jurisdictional claims in published maps and institutional affiliations.

The original article can be found online at https://doi.org/10.1007/s10623-021-00886-4.

\footnotetext{
Chang-An Zhao

zhaochan3@mail.sysu.edu.cn

Ruikai Chen

chen_rk@outlook.com

Sihem Mesnager

smesnager@univ-paris8.fr
}

1 School of Mathematics, Sun Yat-sen University, Guangzhou 510275, People's Republic of China

2 Department of Mathematics, University of Paris VIII, 93526 Saint-Denis, France

3 University Sorbonne Paris Cité, Laboratory Analysis, Geometry and Applications (LAGA), UMR 7539, CNRS, 93430 Villetaneuse, France

4 Telecom Paris, 91120 Palaiseau, France

5 State Key Laboratory of Information Security, Institute of Information Engineering, Chinese Academy of Sciences, Beijing 100093, People's Republic of China

6 Guangdong Key Laboratory of Information Security, Guangzhou 510006, People’s Republic of China 